\title{
Diagnostic Dilemma: Presenting as A Posterior Neck Swelling
}

\author{
Islam MA ${ }^{1 *}$, Chowdhury $\mathrm{NH}^{2}$, Mohammad $\mathrm{T}^{3}$, Mamun $\mathrm{TB}^{4}$, Khan $\mathrm{SR}^{5}$, Rahman ASML ${ }^{6}$ and Mostofa Kamal \\ Arefin $^{7}$
}

${ }^{1}$ Professor \& Head, ENT, Bangladesh Medical college Hospital, Bangladesh

${ }^{2}$ Assistant registrar, ENT, Bangladesh medical college Hospital, Bangladesh

${ }^{3}$ Assistant registrar, ENT, Bangladesh medical college Hospital, Bangladesh

${ }^{4}$ Assistant registrar, ENT, Bangladesh medical college Hospital, Bangladesh

${ }^{5}$ Assistant registrar, ENT, National institute of ENT, Bangladesh

${ }^{6}$ Resident Surgeon, ENT, Bangladesh medical college Hospital, Bangladesh

${ }^{7}$ Otolaryngologist, Dhaka Medical College Hospital, Bangladesh

*Corresponding author: Md Ashraful Islam, Professor \& Head Dept of ENT-Head \&

Neck surgery, Bangladesh Medical college Hospital, Dhaka, Bangladesh.

Received Date: November 04, 2020

Published Date: November 13, 2020

\begin{abstract}
Neck swelling is a common presentation in the general population and has a number of possible causes. Lymph nodes are the commonest among all. Schwannomas are benign slow growing tumors that arise from the Schwann cells of nerve, also called neurilemmoma. Head and Neck schwannomas usually present as solitary with well demarcated lesions. These tumors usually remain asymptomatic but present as slowly enlarging neck masses. Its origin is only determined during surgical procedure along the course and distribution of the nerve. Preoperative diagnosis is usually made by physical examination and aided by ultrasonography, magnetic resonance imaging or computed tomography and fine needle aspiration cytology but open biopsy is not recommended. The main stay of treatment is complete intracapsular excision with preserving the nerve of origin.

Keywords: Schwannomas; Benign tumors; Lateral neck mass
\end{abstract}

\section{Introduction}

Neck masses are often seen in clinical practice, and the otolaryngologists should be able to determine the etiology of a mass using organized, efficient diagnostic methods. Schwannomas are benign encapsulated nerve sheath tumors composed of Schwann cells encompassing motor and sensory peripheral nerves. Embryologically, Schwann cells arise during the fourth week of development from a specialized population of ectomesenchymal cells of the neural crest. Schwann cells form a thin barrier around each extracranial nerve fiber and wrap larger fibers with an insulating myelin sheath to enhance nerve conduction. Schwannoma may be of different variants such as classic, cellular, plexiform, epithelioid, ancient and melanotic. Head and neck schwannomas usually present about $25 \%$ to $45 \%$ and involve cranial nerves V, VII, IX, X, XI and XII, sympathetic chain, brachial or cervical plexus [1]. Schwannomas are generally slow-growing neoplasms with annual tumor growth rates of $3 \mathrm{~mm}$. and its malignant transformation is rare [2].

\section{Case Report}

A 38- year-old female patient presented to us with a slow growing mass on the left side of back of the upper neck for 5 years and pain in the swelling for 2 years. 5 years ago, she noticed a just palpable swelling at the back of neck that was gradually increasing 
in size. It didn't disturb her for 3 years with the swelling, but since then she started mild local pain especially on neck movement. She did not experience any weakness, numbness or loss of function of the left upper limb. The patient had no dysphagia, voice change, hemoptysis, fever, cough or nasal bleeding. No history of recent weight loss or anorexia.

Her general examination was essentially normal. Local examination revealed a diffuse ill-defined swelling in left posterior neck measuring $6 \times 4 \mathrm{~cm}$, extending from the hairline near to root of neck, $2 \mathrm{~cm}$ lateral to midline just deep to posterior border of SCM. On palpation the swelling was oval shaped with ill-defined border, non-tender, and no local rise of temperature. The mass was firm to hard in consistency, immobile but free from overlying skin. It extended under the sternocleidomastoid muscle towards the anterior triangle of neck which was difficult to assess (Figure 1). On flexion of neck, the swelling tends to become more ill-defined that was suggestive of deeper relation to trapezius. Motor and sensory function of left hand was intact. Peripheral pulse was normal. The rest of the neck was normal on palpation (Figure 1). Ultrasound examination of the neck revealed a well-defined cystic lesion deep to trapezius muscle. Fine needle aspiration cytology was suggestive of benign cystic lesion. In January 2020, computed tomography (CT) scanning of the neck was performed and showed a welldefined mass in the posterior triangle, not involving the intra-spinal muscle measuring $4.4 \times 3.1 \times 4.4 \mathrm{~cm}$. CT was in favor of mildly thick wall loculated mixed density cystic lesion at left postero-lateral C1 to C3 spine, possibilities are cystic lymphangioma/epidermoid (Figure 2).

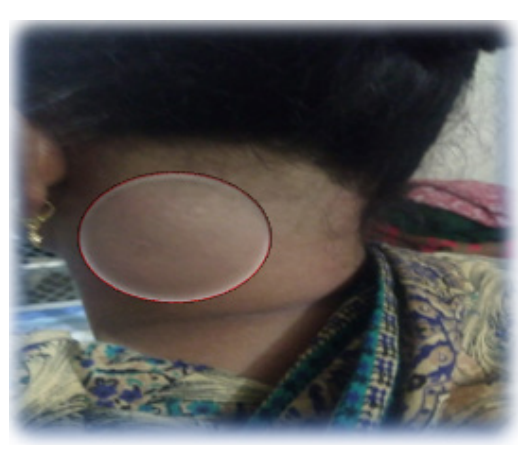

Figure 1: oval demarcated area representing site of swelling in posterior neck.

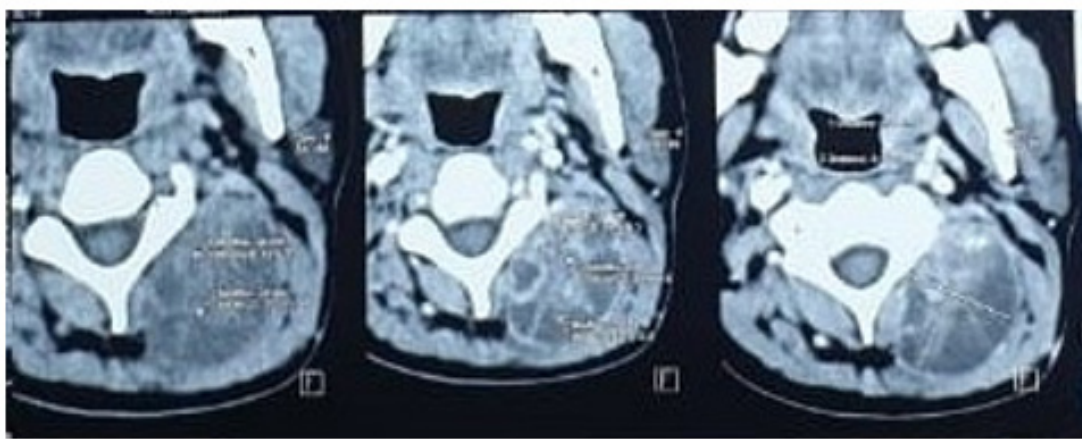

a.

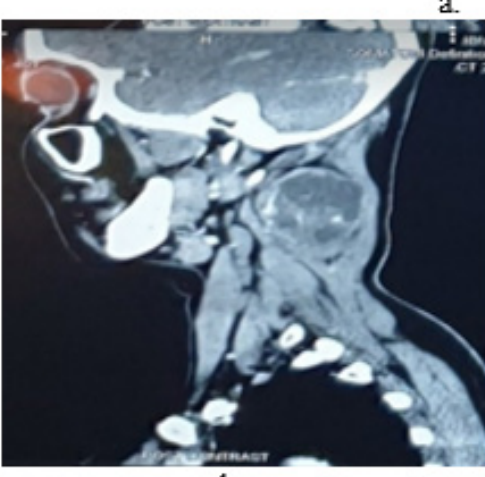

b.

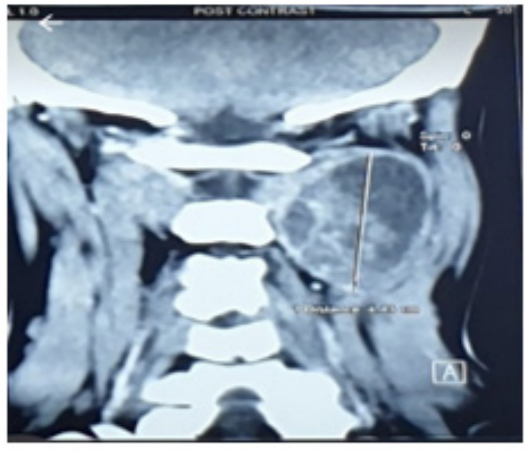

Figure 2: Contrast CT of neck with multiple axial (a), sagittal (b) and coronal (c) views showed well circumscribed oval, non-enhancing mixed density lesion lateral to body of upper 3rd cervical vertebra and deep to trapezius muscle. 
The patient underwent surgery under general anesthesia through left transcervical approach. On exploration of the neck, revealed the mass which went up to the vascular triangle but not adherent to the carotid or internal jugular vein. It was found over the cervical plexus and was on relation to C2 and C3 nerve. The mass was oval shaped, well-circumscribed extended deep to the trapezius muscle and lateral to posterior paravertebral neck muscle up to transverse process of upper 3 cervical vertebra. Anteriorly it was found deep to sternocleidomastoid muscle. The whole mass was carefully separated from attached nerves and excised completely that measured about $4.0 \times 4.0 \times 5.0 \mathrm{~cm}$ (Figure 3). The histopathologic diagnosis was schwannoma and the tumor was composed of spindle shaped cells arranged in interlacing fascicles with nuclear palisading. Focal pseudocystic areas and foamy histiocytes were also found, as well as hyalinized blood vessels. There was no nuclear atypia. There was no sign of damage to the motor fibres.

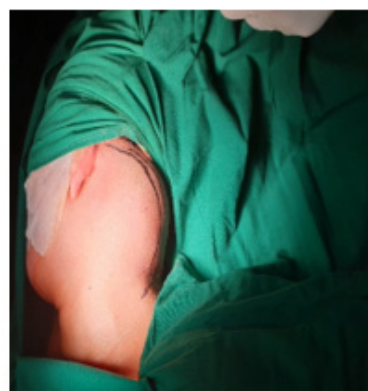

a.

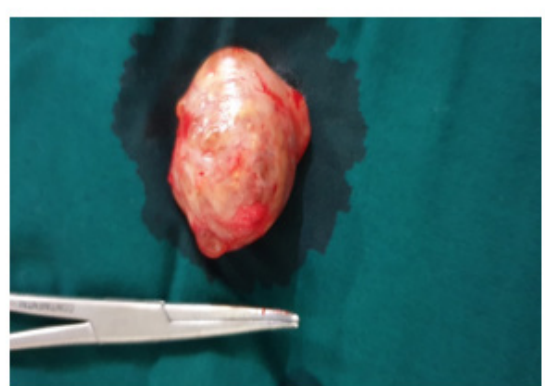

b.

Figure 3: preoperative incision line from tip of mastoid to anterior border of trapezius (a). specimen after excision (b).

\section{Discussion}

Schwannoma, neurofibroma or neuroma are the benign nerve sheath tumor arising from the Schwann cell. Amongst all the schwannomas, head neck schwannomas comprising $25 \%$ to $45 \%$, presenting mostly in the parapharyngeal space [3]. Cystic change is uncommon in these tumors, being reported in only $4 \%$ cases of schwannoma [4]. Rarely, they arise near the vertebral foramina presenting with intraspinal and extraspinal components [5]. Schwannoma arising from cervical plexus are very rare. There are very few literatures on it. The present reported case was cystic found on ultrasonography and fine needle aspiration cytology.

Schwannoma usually compress the nerve fibers if it displays over the tumor capsule with the gradual increasing of tumor and often nerve paralysis may occur preoperatively. Basically, symptomatic presentation depends on the nerve of involvement e.g. vagal schwannoma that is typically characterized by dysphagia and hoarseness, sympathetic schwannoma presented by Horner's syndrome. In most cases, years together patients may become asymptomatic, thus it is difficult to identify the neurological origin based on the physical examination [6]. This case exactly became asymptomatic for years together and symptoms develop after 8 years.

It is difficult to diagnose schwannomas clinically, preoperative diagnosis required radiographic studies, best evaluated by magnetic resonance imaging (MRI) or computed tomography (CT). On non-contrast CT, schwannomas present hypodense in comparison to surrounding muscle. Contrast enhancement revealed homogeneously solid or heterogeneous mass. The diagnostic difficulty become obvious when it undergo cystic changes. Our case presented with cystic presentation which increased than non-cystic schwannomas. The cystic change has been attributed to necrosis, mucinous degeneration, and hemorrhage; and microcysts formation [7]. Fine-needle aspiration cytology (FNAC) in the diagnosis of schwannomas remains debatable [7] which was supportive in our case as the finding was in favor of cystic lesion.

Microscopically, schwannomas are usually remain encapsulated and may present as solid or cystic masses. The present dissected tissue was composed of spindle shaped cells arranged in interlacing fascicles with nuclear palisading. They are composed of densely arranged with spindle-shaped Schwann cells with areas of palisading nuclei, verocay bodies known as Antony type A and Antony B is characterized by a hypo cellular arrangement and a large quantity of myxoid tissue which was relevant in the published literature [8]. In Immunochemestry S-100 protein is a neural crest marker antigen is an important diagnostic tool, as schwannomas show intense immunostaining for S-1001 but in our case this test was not done.

Small tumors are usually present as uninodular but larger tumors may be multinodular with degenerative features including cyst formation, fibrosis and calcification which was similar in our patient. MRI is the best modality in the pre-operative work-up as it is helpful in defining diagnosis [9] and in evaluating the extent and the relationship of the tumor with the jugular vein and the carotid artery but CT with contrast was done in our case. 


\section{Conclusion}

It is difficult to diagnose a cervical plexus schwannoma by routine clinical examination. MRI plays the most important, although CT is a good alternative. FNAC also should be done to rule out the cytological nature of the lesion, though it has little rule on diagnosis of cystic schwannoma. The treatment is surgical. Intracapsular excision is the choice of treatment with preserving the nerve of origin. A cystic lesion on posterior neck have very limited differential diagnosis. Planned imaging, well planned surgical excision and last but not the least is its histopathological confirmation completes the journey.

\section{Acknowledgement}

None

\section{Conflict of Interest}

None.

\section{References}

1. Ishtyaque Ansari, Ashfaque Ansari, Arjun Antony Graison, Anuradha J Patil, Hitendra Joshi (2018) Head and Neck Schwannomas: A Surgical Challenge-A Series of 5 Cases. Case Rep Otolaryngol.
2. Anastasios K (2009) Schwannomas of the head and neck. Oncol Rev 3: 107-111.

3. Chang SC, Schi YM (1984) Neurilemmoma of the vagus nerve. A case report and brief literature review. Laryngoscope 94(7): 946-949.

4. Wakoh M, H Yonezu, T Otonari, T Sano, K Matsuzaka, et al. (2005) Two cases of schwannoma with marked cystic changes. Dentomaxillofac Radiol 34(1): 44-50.

5. P Iacconi, M Faggioni, C De Bartolomeis, C Iacconi, C Caldarelli (2012) cervical sympathetic chain schwannoma: a case report. Acta otorhinolaryngol Ital 32(2): 133-136.

6. De Araujo CE, Daniel M Ramos, Raquel A Moyses, Marcelo D Durazzo, Cláudio R Cernea, et al. (2008) Neck nerve trunks schwannomas: clinical featuresand postoperative neurologic outcome. Laryngoscope 118: 1579-1582.

7. Luka V, Zoran Lajtman, Josip Mihalj, Jasmina Plašćak, Darija Mahović Lakušić, et al. (2018) Brachial Plexus Schwannoma-Case Report and Literature Review. Acta Clin Croat 57: 366-371.

8. Nilesh c, et al. (2014) A rare Cervical Nerve Root, C2-C3 Schwannoma. Otolaryngology online journal 4(2).

9. Rohaizam J, Ing Ping Tang, Doris Evelyn Yah Hui Jong, Tee Yong Tan, Mohammad Zulkarnaen Ahmad Narihan (2012) Cervical Schwannoma: Report of Four Cases. Med J Malaysia 67(3): 345-346. 